PROCEEDINGS OF THE

AMERICAN MATHEMATICAL SOCIETY

Volume 140, Number 7, July 2012, Pages 2441-2446

S 0002-9939(2011)11109-2

Article electronically published on November 17, 2011

\title{
AN EXTREMAL DECOMPOSITION PROBLEM FOR HARMONIC MEASURE
}

\author{
VLADIMIR N. DUBININ AND MATTI VUORINEN
}

(Communicated by Mario Bonk)

\begin{abstract}
Let $E$ be a continuum in the closed unit disk $|z| \leq 1$ of the complex $z$-plane which divides the open disk $|z|<1$ into $n \geq 2$ pairwise nonintersecting simply connected domains $D_{k}$ such that each of the domains $D_{k}$ contains some point $a_{k}$ on a prescribed circle $|z|=\rho, 0<\rho<1, k=1, \ldots, n$. It is shown that for some increasing function $\Psi$, independent of $E$ and the choice of the points $a_{k}$, the mean value of the harmonic measures

$$
\Psi^{-1}\left[\frac{1}{n} \sum_{k=1}^{k} \Psi\left(\omega\left(a_{k}, E, D_{k}\right)\right)\right]
$$

is greater than or equal to the harmonic measure $\omega\left(\rho, E^{*}, D^{*}\right)$, where $E^{*}=$ $\left\{z: z^{n} \in[-1,0]\right\}$ and $D^{*}=\{z:|z|<1,|\arg z|<\pi / n\}$. This implies, for instance, a solution to a problem of R. W. Barnard, L. Cole, and A. Yu. Solynin concerning a lower estimate of the quantity $\inf _{E} \max _{k=1, \ldots, n} \omega\left(a_{k}, E, D_{k}\right)$ for arbitrary points of the circle $|z|=\rho$. These authors stated this hypothesis in the particular case when the points are equally distributed on the circle $|z|=\rho$.
\end{abstract}

\section{INTRODUCTION AND FORMULATION OF THE RESULT}

Problems concerning extremal decomposition of domains on the complex sphere go back to the works of Lavrentiev, Goluzin, Nehari, Jenkins and have a rich history and are more or less directly associated with several areas of geometric function theory (see, e.g., [J2, Kuz, $[\mathrm{S}]$ ). A majority of extremal decompositions is related to estimates for the products whose factors are powers of inner radii of pairwise nonoverlapping domains. For the case of harmonic measure the problem concerning extremal decomposition was perhaps first formulated in the paper of R.W. Barnard, L. Cole, and A. Yu. Solynin BCS]. Let $E$ be a continuum in the closed unit disk $\bar{U}, U=\{z:|z|<1\}$, and let it divide $U$ into two subdomains $D_{1} \ni \rho$ and $D_{2} \ni-\rho$, $0<\rho<1$. Thus $U \backslash E=D_{1} \cup D_{2}$. The authors of the paper [BCS] give the minimum of the sums

$$
\frac{1}{2}\left(\omega\left(\rho, E, D_{1}\right)+\omega\left(-\rho, E, D_{2}\right)\right),
$$

Received by the editors December 4, 2010 and, in revised form, January 6, 2011 and February $24,2011$.

2010 Mathematics Subject Classification. Primary 30C85.

Key words and phrases. Harmonic measure, inner radius, extremal decomposition.

The research of the first author was supported by the Far-Eastern Branch of the Russian Academy of Sciences, project 09-III-A-01-007.

The second author was supported by the Academy of Finland, project 2600066611.

(C)2011 American Mathematical Society Reverts to public domain 28 years from publication 
taken over the set of all continua $E \subset \bar{U}$ that divide $U$ into a pair of domains as described above. Here $\omega(z, E, D)$ denotes the harmonic measure of the set $E \cap \partial D$ with respect to the domain $D$, evaluated at the point $z$. For the properties of the harmonic measure we refer to [A, Ch 1.1]. In the paper [BCS] also a physical motivation of the problem is given. It is of interest to observe that the extremal configuration in this problem is not symmetric with respect to the imaginary axis. Among other things the authors of the paper $[\mathrm{BCS}$ discuss the problem of finding the lower bound

$$
\inf _{E} \max _{k=1, \ldots, n} \omega\left(a_{k}^{*}, E, D_{k}\right)
$$

over all continua $E$ that divide $U$ into $n$ simply connected domains

$$
D_{k} \ni a_{k}^{*}=\rho \exp (2 \pi i(k-1) / n), \quad k=1, \ldots, n, n \geq 3 .
$$

They conjectured that the extremal configuration of the minmax problem consists of $n$ circular sectors

$$
D_{k}^{*}=\{z \in U:|\arg z-2 \pi(k-1) / n|<\pi / n\}, \quad k=1, \ldots, n,
$$

and observed that under the additional assumption that the closure of $\partial D_{k} \cap U$ is connected for all $k=1, \ldots, n$, this can be proved applying Jenkins's theory of extremal decomposition [J1, S] (see [BCS, p. 246]). A. Yu. Solynin has informed the authors that this result has remained unpublished.

In this paper we prove the following theorem.

Theorem 1.1. For a given $\rho, 0<\rho<1$, let $E$ be a continuum in the closed unit disk $\bar{U}$ dividing the open disk $U$ into $n \geq 2$ pairwise nonintersecting simply connected domains $D_{k}$, such that each of the domains $D_{k}$ intersects the circle $|z|=$ $\rho, k=1, \ldots, n$. Then for arbitrary points $a_{k} \in D_{k}$ on the circle $|z|=\rho$, the following inequality holds:

$$
\frac{1}{n} \sum_{k=1}^{n} \log \frac{1+\sin \left(\pi \omega_{k} / 2\right)}{1-\sin \left(\pi \omega_{k} / 2\right)} \geq-n \log \rho
$$

where $\omega_{k}=\omega\left(a_{k}, E, D_{k}\right), k=1, \ldots, n$. Equality holds in (1.2) if and only if

$$
E=\left\{z:\left(e^{i \theta} z\right)^{n} \in[-1,0]\right\}, D_{k}=\left\{z: z e^{i \theta} \in D_{k}^{*}\right\}
$$

and $a_{k}=a_{k}^{*} e^{-i \theta}, k=1, \ldots, n$, for some fixed real $\theta$.

Because the function

$$
\Psi(x)=\log \frac{1+\sin (\pi x / 2)}{1-\sin (\pi x / 2)}
$$

is strictly increasing on the interval $(0,1)$, the inequality (1.2) implies a solution to the problem of R. W. Barnard, L. Cole, and A. Yu. Solynin [BCS], and furthermore, the points $a_{k}$ need not coincide with $a_{k}^{*}, k=1, \ldots, n$. Our proof is based on the result of the first author concerning the product of inner radii of nonoverlapping domains with respect to "free" points on the given circle [D1]. We also make use of an idea of J. Krzyż about the transition from inner radius to conformal invariants $[\mathrm{K}]$. If in the case of $[\mathrm{K}]$ the Green function was discussed, then in our case the harmonic measure is in focus. We note further that by the invariance of the harmonic measure under Möbius automorphisms of the disk $U$, the inequality (1.2) holds for all points $a_{k}$, located on an arbitrary hyperbolic circle in $U$ of hyperbolic radius $\log ((1+\rho) /(1-\rho))$. 


\section{Proof of Theorem 1.1}

Fix an integer $k, 1 \leq k \leq n$. If all the boundary points of the domain $D_{k}$ lie in the continuum $E$, then $\omega_{k}=1$ and the left side of the inequality (1.2) is $+\infty$. In what follows we exclude this case for all $k=1, \ldots, n$. Now let $z$ be some point of the boundary $\partial D_{k}$, not contained in $E$. Then $z \in T:=\partial U$. In fact, in the opposite case, $z \in U \backslash E$, and therefore $z \in D_{j}$ for some $j, 1 \leq j \leq n$. Because of the openness of a domain, we see that $D_{j} \cap D_{k} \neq \emptyset$, which contradicts the hypothesis of the theorem. Further, because the set $E$ is closed, on the circle $T$ there is an open arc $\alpha$ containing the point $z$ and not containing any points of $E$. We prove that all points of this arc are boundary points of the domain $D_{k}$. Suppose that there exists a point $z^{\prime} \in \alpha \backslash \partial D_{k}$. Because $z^{\prime} \notin E$, there exists a domain $D_{k^{\prime}}$ such that $z^{\prime} \in$ $\partial D_{k^{\prime}}, 1 \leq k^{\prime} \leq n$. Choose sequences of points $z_{m}, z_{m}^{\prime}, m=1,2, \ldots$, and circular arcs $\lambda_{m}, m=1,2, \ldots$, in $U$ with $z_{m}, z_{m}^{\prime}$ as end points and satisfying the following condition. The points $z_{m} \in D_{k}, z_{m} \rightarrow z, m \rightarrow \infty ; z_{m}^{\prime} \in D_{k^{\prime}}, z_{m}^{\prime} \rightarrow z^{\prime}, m \rightarrow \infty$, and the $\operatorname{arcs} \lambda_{m} \rightarrow T$ when $m \rightarrow \infty$. This last convergence means that the upper bound of the distances of the points of the arc $\lambda_{m}$ to the circle $T$ approaches 0 when $m \rightarrow \infty$. Because $D_{k} \cap D_{k^{\prime}}=\emptyset$, we see that on every arc $\lambda_{m}$ there exists a point $e_{m} \in E, m=1,2,3, \ldots$ The sequence of the points $\left\{e_{m}\right\}_{m=1}^{\infty}$ contains a subsequence converging to a point $e$ of the $\operatorname{arc} \alpha$. Because $E$ is closed, the point $e$ must be in $\alpha \cap E$, a contradiction to the choice of $\alpha$. Let us denote by $\alpha_{k}$ the maximal open subarc of the circle $T$, containing the $\operatorname{arc} \alpha$ and not containing the points of the set $E$. Clearly, the end points of the $\operatorname{arc} \alpha_{k}$ are contained in $E$. The domain $D_{k}$ cannot contain on its boundary two different arcs of the aforementioned type (each corresponding to different points $z$ ) in view of the connectedness of the set $E$. We see that $\alpha_{k}=\left(\partial D_{k}\right) \backslash E, k=1,2, \ldots, n$.

We introduce the notation $B_{k}=D_{k} \cup \alpha_{k} \cup\left\{z: 1 / \bar{z} \in D_{k}\right\}, k=1, \ldots, n$. From what was said above it follows that the sets $B_{k}, k=1, \ldots, n$, are simply connected pairwise nonintersecting domains on the Riemann sphere $\overline{\mathbb{C}}$. By Riemann's theorem, there exists a function $\varphi_{k}$ mapping conformally and univalently the corresponding domain $B_{k}$ onto the domain $G_{k}$ which is the complement of the $\operatorname{arc} \beta_{k}:=\{w:|w|=$ $\left.1,|\arg w| \leq \theta_{k}\right\}, \varphi_{k}\left(a_{k}\right)=0, \varphi_{k}^{\prime}\left(a_{k}\right)>0, k=1, \ldots, n$. On the basis of symmetry, the image of the domain $D_{k}$ is the disk $|w|<1$, and further $\varphi_{k}\left(\alpha_{k}\right)=\left\{w \in G_{k}\right.$ : $|w|=1\}$ and $\varphi_{k}\left(E \cap \partial D_{k}\right)=\beta_{k}$. Because the harmonic measure is conformally


Figure 1. Left: The continuum $E$ divides the unit disk $U$ into three domains. Right: The image of the domain $D_{k}$ under $\varphi_{k}$. 
invariant, we have

$$
\omega_{k}=\omega\left(0, \beta_{k}, U_{w}\right)=\theta_{k} / \pi, \quad k=1, \ldots, n .
$$

We denote by $\ell_{k}$ the union of a finite number of half-open arcs of the analytic curve $\gamma_{k}:=\varphi_{k}^{-1}((-1,0])$, having the following property: for every $r, \rho \leq r<1$, the circle $|z|=r$ intersects with $\ell_{k}$ only at one point. We set $z_{k}(r)=\left\{z \in \ell_{k}:|z|=r\right\}$. The construction of the $\operatorname{arcs} \ell_{k}$ can be made in several ways. We choose one of them. It follows from the paper [D1] (cf. also [D2, p. 53]) that for every $r, \rho \leq r<1$, the inequality

$$
\sqrt[n]{\Pi_{k=1}^{n} r\left(B_{k}, z_{k}(r)\right)} \leq \frac{4 r}{n}
$$

holds and furthermore the equality in (2.1) holds if and only if the domains $B_{k}$ and the points $z_{k}(r)$ coincide with the domains $\{z:|\arg z-2 \pi(k-1) / n|<\pi / n\}$ and the points $r \exp (2 \pi(k-1) / n)$, respectively, with a possible rotation around the origin. Here $r(B, z)$ stands for the inner radius of the domain $B$ with respect to a point $z \in B$; see [D2, Sec. 1]. The inequality (2.1) was proved by the piecewise separating symmetrization method of [D2].

By the arithmetic-geometric mean inequality this implies that

$$
\frac{1}{n} \sum_{k=1}^{n} \frac{1}{r\left(B_{k}, z_{k}(r)\right)} \geq \frac{n}{4 r} \text {. }
$$

Integration yields the conclusion that

$$
\begin{aligned}
& \frac{1}{n} \sum_{k=1}^{n} \int_{\gamma_{k}} \frac{|d z|}{r\left(B_{k}, z\right)} \geq \frac{1}{n} \sum_{k=1}^{n} \int_{\ell_{k}} \frac{|d z|}{r\left(B_{k}, z\right)} \\
& \quad \geq \frac{1}{n} \sum_{k=1}^{n} \int_{\rho}^{1} \frac{d r}{r\left(B_{k}, z_{k}(r)\right)} \geq \int_{\rho}^{1} \frac{n}{4 r} d r=-\frac{n}{4} \log \rho .
\end{aligned}
$$

On the other hand

$$
\int_{\gamma_{k}} \frac{|d z|}{r\left(B_{k}, z\right)}=\int_{-1}^{0} \frac{d w}{r\left(G_{k}, w\right)}
$$

for all $k=1, \ldots, n$. For the computation of the inner radius $r\left(G_{k}, w\right)$ we map the domain $G_{k}$ by a univalent conformal mapping onto the unit disk $|\zeta|<1$. It is easy to see that for such a map we can take a superposition of the following functions:

$$
w_{1}=\frac{w-1}{w+1}, \quad w_{2}=-i w_{1} \operatorname{ctg} \frac{\theta_{k}}{2}, \quad \zeta=w_{2}-\sqrt{w_{2}^{2}-1},
$$

where the last mapping is that branch of the function, inverse of the Joukowski transformation, which maps $\infty$ to zero. Because under a conformal univalent mapping the inner radius is multiplied by the modulus of the derivative at the corresponding point, we have

$$
r\left(G_{k}, w\right)\left|w_{1}^{\prime}(w) \operatorname{ctg} \frac{\theta_{k}}{2} \zeta^{\prime}\left(w_{2}\right)\right|=r\left(U_{\zeta}, \zeta\right)=1-|\zeta|^{2} .
$$

Simple calculations give from this

$$
r\left(G_{k}, w\right)=\frac{1-w}{\sin \left(\theta_{k} / 2\right)} \sqrt{w^{2}-2 w \cos \theta_{k}+1} .
$$


Inserting this value of the inner radius in the inequality (2.3), we obtain

$$
\int_{-1}^{0} \frac{d w}{r\left(G_{k}, w\right)}=\sin \frac{\theta_{k}}{2} \int_{-1}^{0} \frac{d w}{(1-w) \sqrt{w^{2}-2 w \cos \theta_{k}+1}}=\frac{1}{4} \log \frac{1+\sin \left(\theta_{k} / 2\right)}{1-\sin \left(\theta_{k} / 2\right)} .
$$

The computation of the integral is carried out either with standard integration methods or using tables such as [PBM, p. 94]. Applying the relations (2.2) and (2.3) we arrive at the desired inequality (1.2) .

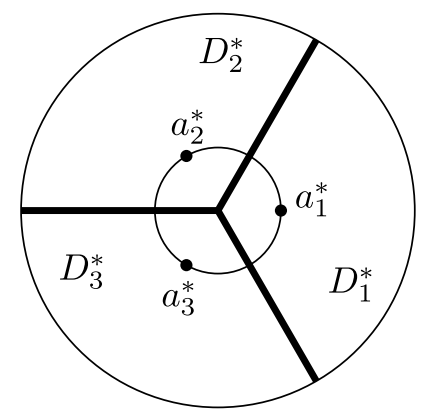

FiguRE 2. Extremal configuration for $n=3$.

We now assume that in the inequality (1.2) we have equality. Then the equalities also hold in (2.2) and (2.1) for all $r, \rho \leq r<1$. From the equality in (2.2) it follows that the curves $\ell_{k}=\gamma_{k}$ are segments on radial rays, emanating from the origin, $k=1, \ldots, n$. Equality in (2.1) shows that the angles between neighboring rays are equal to $2 \pi / n$ and the domains $B_{k}$ are sectors with angle $2 \pi / n$ for which these rays are bisectors. This observation implies that for some real $\theta, a_{k}=a_{k}^{*} e^{-i \theta}$ and $D_{k}=\left\{z: z e^{i \theta} \in D_{k}^{*}\right\}, k=1, \ldots, n$. In this case the continuum $E$ necessarily contains the set $E_{\theta}:=\left\{z:\left(z e^{i \theta}\right)^{n} \in[-1,0]\right\}$. With a simple verification we see that for $E=E_{\theta}$ in (1.2) we have the sign of equality. It remains to observe that for other continua $E \supset E_{\theta}, E \neq E_{\theta}$ we have a strict inequality. The theorem is proved.

\section{ACKNOWLEDGEMENTS}

This work was completed during the visit of the first author to the University of Turku, Finland. The authors are indebted to the referee, whose comments were valuable.

\section{REFERENCES}

[A] L.V. Ahlfors, Conformal invariants: topics in geometric function theory. McGraw-Hill Series in Higher Mathematics. McGraw-Hill Book Co., New York-Düsseldorf-Johannesburg, 1973. MR0357743 (50:10211)

[BCS] R.W. Barnard, L. Cole, and A. Yu. Solynin, Minimal harmonic measure on complementary regions. Comput. Methods Funct. Theory 2 (2002), 229-247. MR2000559 (2004g:30037)

[D1] V.N. Dubinin, The product of inner radii of "partially nonoverlapping" domains. Questions in the metric theory of mappings and its application (Proc. Fifth Colloq. Quasiconformal Mappings, Generalizations Appl., Donetsk, 1976) (Russian), 24-31, "Naukova Dumka", Kiev, 1978. MR558368(81e:30029)

[D2] V.N. Dubinin, Symmetrization in the geometric theory of functions of a complex variable. Uspekhi Mat. Nauk 49 (1994), no. 1(295), 3-76 (Russian); translation in Russian Math. Surveys 49 (1994), no. 1, 1-79. MR1307130 (96b:30054) 
[J1] J.A. Jenkins, On the existence of certain general extremal metrics. Ann. of Math. (2) 66 (1957), 440-453. MR0090648 (19:845g)

[J2] J.A. Jenkins, The method of the extremal metric (English summary). Handbook of complex analysis: geometric function theory, Vol. 1, 393-456, ed. by R. Kühnau, NorthHolland, Amsterdam, 2002. MR.1966200 (2004a:30024)

[K] J. Krzyż, Circular symmetrization and Green's function (Russian summary). Bull. Acad. Polon. Sci. Ser. Sci. Math. Astr. Phys. 7 (1959), 327-330. MR0107701 (21:6424)

[Kuz] G. V. Kuz'mina, Methods of the geometric theory of functions. II. Algebra i Analiz 9:5 (1997), 1-50 (Russian); translation in St. Petersburg Mathematical Journal, 1998, 9:5, 889-930. MR 1604397(99c:30047a)

$[\mathrm{PBM}]$ A.P. Prudnikov, Yu. A. Brychkov, and O. I. Marichev, Integraly i ryady [Integrals and series]. Elementarnye funktsii [Elementary functions] (Russian), "Nauka", Moscow, 1981, 799 pp. MR635931 (83b:00009)

[S] A. Yu. Solynin, Moduli and extremal metric problems. Algebra i Analiz 11 (1999), no. 1, 3-86 (Russian); translation in St. Petersburg Math. J. 11 (2000), no. 1, 1-65. MR.1691080 (2001b:30058)

Institute of Applied Mathematics, Far-Eastern Branch of the Russian Academy of Sciences, Vladivostok, Russia

E-mail address: dubinin@iam.dvo.ru

Department of Mathematics, University of Turku, Turku 20014, Finland

E-mail address: vuorinen@utu.fi 